\title{
Dramaturgia Andaluza Contemporánea
}

La Generación Romero Esteo

María Jesús Bajo Martínez

\section{OpenEdition}

\section{Journals}

Edición electrónica

URL: https://journals.openedition.org/cher/1032

DOI: $10.4000 /$ cher. 1032

ISSN: 2803-5992

Editor

Presses universitaires de Strasbourg

\section{Edición impresa}

Fecha de publicación: 11 julio 2019

Paginación: 11-19

ISBN: 979-10-344-0046-1

ISSN: 1968-035X

Referencia electrónica

María Jesús Bajo Martínez, «Dramaturgia Andaluza Contemporánea», reCHERches [En línea], 22 | 2019,

Publicado el 07 octubre 2021, consultado el 17 noviembre 2021. URL: http://journals.openedition.org/ cher/1032 ; DOI: https://doi.org/10.4000/cher.1032 


\title{
Dramaturgia Andaluza Contemporánea La Generación Romero Esteo
}

\author{
María Jesús Bajo Martínez'
}

$\mathrm{E}_{\mathrm{c}}^{\mathrm{n}}$ 1997, el director de escena Emilio Hernández, entonces director del Centro Andaluz de Teatro, puso en marcha el Concurso de Textos Teatrales para Jóvenes Autores Andaluces Premio Romero Esteo, con la colaboración del Centro de Documentación de las Artes Escénicas de Andalucía, ambas entidades dependientes de la Consejería de Cultura de la Junta de Andalucía. Posteriormente continuó esta labor el también director del Centro Andaluz de Teatro, Francisco Ortuño Millán. Pocos proyectos de promoción de la escritura dramática han conseguido resultados tan positivos como este; además de ser una de las iniciativas del Centro Andaluz de Teatro que más frutos ha dado a lo largo de su trayectoria, tanto por la cantidad de autores que surgieron, como por su calidad. El objetivo de este premio era promocionar la escritura dramática entre los jóvenes andaluces. Como explicaba Emilio Hernández en las bases del primer concurso,

[...] se dispone a incentivar el acercamiento al teatro de Jóvenes Autores Andaluces menores de treinta años, que reflejen un punto de vista, una estética y una realidad acorde con una generación que nació con la democracia, creció con la informática y se desarrolla con la televisión digital, y que puede reconocer en el teatro una expresión más libre y la vía más directa de comunicación con sus conciudadanos. ${ }^{2}$

El resultado fue el principio de la carrera de un importante número de autores dramáticos, que actualmente conforman buena parte del panorama escénico andaluz y nacional, habiéndose constituido en claro relevo a la generación anterior de autores andaluces, compuesta entre otros por Antonio Estrada, Alfonso Zurro, Antonio Álamo, Juan Larrondo, Antonio Onetti... Pero

1 María Jesús Bajo Martínez, Centro de Documentación de las Artes Escénicas de Andalucía.

2 Folleto del primer Concurso de Textos Teatrales para Jóvenes Autores Andaluces en 1997. 
además, muchos de ellos forman un grupo compacto con no pocas características comunes y relaciones personales y profesionales.

Han participado en el concurso, obteniendo diferentes galardones, numerosos autores, entre los que destacaremos los siguientes: Tomás Afán, Javier Berger, Juanma Cabañas, Rafael Cobos, Jorge Dubarri, Rafael García Guzmán, Antonio Hernández Centeno, Carlos Herrera Carmona, Gonzalo Lloret Marín, Dámaris Matos, David Montero, José Manuel Mora Ortiz, Gracia Morales, Antonio Miguel Morales Montoro, Miguel Palacios, Carmen Pombero, Antonio Raposo, Antonio Rincón-Cano, Antonio José Rojano, Sergio Rubio, Marilia Samper, Juan Alberto Salvatierra y José Francisco Ortuño. Aunque hay que especificar que no todos están igual de integrados en el grupo, ni mantienen los mismos lazos.

No vamos a polemizar aquí sobre el concepto de generación y su vigencia en el estudio de la literatura, fuertemente arraigado en el primer cuarto del siglo XX, ni a cuestionar las opiniones del pensador José Ortega y Gasset, quien dedicó a este tema diferentes estudios, ni tampoco la influencia que sus ideas tuvieron durante largo tiempo en Europa y América. Lo cierto es que este concepto surge como respuesta para estudiar a los jóvenes como categoría social, ya desde la Antigüedad. Por otra parte, el filósofo Wilhelm Dilthey opina (1994) que una generación parte de un acontecimiento histórico, y que sus miembros han nacido en unos años próximos y se han formado en unas circunstancias vitales semejantes, que propicia una coincidencia de intereses e ideas. Y esta realidad sí se cumple en este grupo. Ya Julius Petersen (1984) establecía en Filosofía de la ciencia literaria. las siguientes condiciones para pertenecer a una generación: haber nacido en años cercanos, poseer una educación similar, mantener relaciones entre los miembros, un acontecimiento o experiencia que los aglutine, tener un mismo lenguaje y contar con un líder, aunque esta última condición no siempre se cumple. Si aplicamos estos criterios a los autores objeto de este estudio, se cumplen en gran medida.

Todos los autores han nacido en una horquilla de fechas que va desde 1968, Tomás Afán, a 1985, Antonio Raposo (solamente se podían presentar al premio los menores de treinta años). En cuanto a su formación, la mayoría de ellos cuenta con una educación escénica muy amplia. Predominan los licenciados en arte dramático, se acrecienta así en relación a la generación anterior la formación en artes escénicas. Muchos de estos autores son también licenciados en comunicación o filología. Este grupo ha colaborado en diversos proyectos y permanecido muy unido, baste recordar las colaboraciones entre Antonio Hernández Centeno y Carmen Pombero tanto en teatro como en televisión, o entre Gracia Morales y Juan Alberto Salvatierra, últimamente en el complejo proyecto La grieta. Estos dos autores y Antonio Hernández Centeno coescribieron el texto dramático Puertas Cerradas. Por su parte, Juan Alberto Salvatierra llevó a escena La reunión, de Miguel Palacios. Javier Berger ha colaborado con José Francisco Ortuño en la realización de guiones para televisión, ha escrito junto a Tomás Afán Políticamente incorrecto, y ha dirigido textos de David Montero, Miguel Palacios, José Francisco Ortuño, Tomás Afán, 
Antonio Hernández Centeno, y en la actualidad trabaja en la puesta en escena de un texto de Juanma Cabañas. Sergio Rubio y Rafa Cobos han colaborado en programas de formación.

Lo que estos creadores cuentan en común es su experiencia al presentarse al premio y haber sido ganadores o finalistas, algunos de ellos en varias ocasiones. Este hecho hizo que sus obras fueran montadas y publicadas, y que pudieran asistir a cursos de perfeccionamiento, que resultaron vitales para su formación. Este importante aspecto ya se contemplaba en el primer folleto anunciador de las bases del concurso:

Por eso con la convocatoria de este primer premio para Jóvenes Autores Andaluces, el Centro Andaluz de Teatro no solo invita a la presentación de unos textos, sino indirectamente a la formación de los jóvenes escritores en Escuelas o Talleres de Dramaturgia, a la lectura y estudio de las raíces de nuestro teatro, y al conocimiento del teatro contemporáneo de nuestro entorno que está suponiendo en varios países europeos uno de los campos más propicios de expresión para la agresividad creadora de la juventud. ${ }^{3}$

Entre otros, impartieron cursos en este marco: Sanchis Sinisterra, David Harrower, Sarah Kane, David Greig, Michel Azamá, Gerardjan Rijnders, Rodrigo García, Rafael Spregelburd y April De Angelis. En Cazorla, el CAT organizó un encuentro de jóvenes dramaturgos europeos en el que participaron José Francisco Ortuño, Javier Berger, Tomás Afán, Gracia Morales, Antonio Hernández Centeno y José Manuel Mora. En lo que concierne a la formación más allá de nuestras fronteras, Gracia Morales fue enviada por el CAT a Pont-àMousson, Francia, para participar en La Mousson d'été, encuentro dedicado al descubrimiento y promoción de nuevas escrituras dramáticas. Miguel Palacios y Juanma Cabañas formaron parte de "Colours of the Chameleon", un encuentro de jóvenes autores europeos organizado por el Traverse Theatre de Edimburgo con el apoyo del British Council. Sergio Rubio, estuvo en el Young Writers de Wiesbaden y en una reunión europea de centros dramáticos que tuvo lugar en Niza, en donde se decidió enviarlo a Salónica para la puesta en escena de uno de sus textos.

La publicación de los textos premiados, y en ocasiones de los finalistas, contribuyó sin duda a dar a conocer y consolidar la entonces incipiente carrera de estos jóvenes autores, como opinaba el propio Miguel Romero Esteo en el prólogo al número cero de la colección:

Bienvenido este editarles obras a los jóvenes autores andaluces, y así abrirles a las obras una puerta para una larga vida que las aleje de quedar en meros textos de consumo literario-teatral, o cosa similar. Mi más cordial enhorabuena al Centro Andaluz de Teatro, por organizar este premio; y al Centro de Documentación de las Artes Escénicas de Andalucía porque, y para bien de todo el teatro andaluz, esto de publicar a jóvenes autores no es precisamente un asunto menor sino que un asunto clave. (Berger 2001, Ortuño 2001, Centeno 2001)

3 Ibídem. 
El Centro de Documentación de las Artes Escénicas de Andalucía con su directora al frente, Lola Vargas-Zúñiga, inició una nueva colección denominada Premio Miguel Romero Esteo para la publicación de los textos ganadores, y en ocasiones los accésit. El número cero fue publicado en 2001, junto con los títulos premiados de las ediciones de 1997, 1998 y 1999, en un formato original y moderno, cuya cubierta era siempre igual, con la salvedad del color. Se pensó en un precio muy asequible, 3 euros, para facilitar su acceso al público joven. Posteriormente se editaron seis números más, conforme se fallaban nuevos premios. Cada texto cuenta con una breveintroducción orientativa sobre el mismo ${ }^{4}$.

La primera convocatoria sorprendió por el extraordinario recibimiento que tuvo, alrededor de cien textos. Igualmente sorprendió la modernidad y frescura de algunos de ellos. Y ese era el objetivo que se pretendía conseguir desde el primer momento, como ya indicaba la elección del autor que daba nombre al propio premio, Miguel Romero Esteo, un escritor heterodoxo y transgresor, de imaginación ubérrima y épica.

Estos autores poseían ya desde sus inicios unas características compartidas que se han ido manteniendo, y como indicaba en 2011 Eduardo Pérez-Rasilla, en relación a los nuevos autores españoles, "Por lo general, su escritura se muestra depurada y suele responder a una elaboración cuidada y precisa, llamativamente ambiciosa en lo que a los aspectos formales se refiere" (Pérez-Rasilla 2011: 14). Efectivamente estos creadores a lo largo de su trayectoria, se caracterizan por la modernidad formal y el tratamiento de los temas que hacen en sus obras, como las drogas o la homosexualidad, que no solo hacen suyos con absoluta naturalidad, sino que tratan dentro de la mayor cotidianeidad (temas que ya aparecieron en la dramaturgia inmediatamente anterior). El yo, el ser humano en su soledad está patente, así como sus insatisfacciones, buen ejemplo de esto es la pieza de Marilia Samper Menú del día. Las relaciones personales, tanto sentimentales como de poder, subyacen en gran parte de las historias, como Over the rainbow, de Antonio Rincón-Cano.

Otro tema muy presente a través de los años es la relación de pareja y el tiempo destructor, como se puede apreciar en Hasta que la muerte. Degeneración en nueve escenas, donde, como su título indica, Gonzalo Lloret Marín muestra la degeneración en el tiempo de la relación de una pareja de forma progresiva y destructiva, o la lacerante incomunicación en Silencio, de Sergio Rubio. La soledad del individuo moderno y la deshumanizada gran ciudad también ocupan su lugar en esta dramaturgia que, aunque nace en los últimos años del siglo XX, es plenamente del siglo XXI, y se ve perfectamente representada en las breves historias de Piezas urbanas, de Carmen Pombero. Igualmente se ocupan del cambio en la familia y los nuevos modelos. Pero si hay alguna temática común a todos ellos es la actualidad social más inmediata. Esto sucedió ya desde las primeras convocatorias, donde se reflejaba la entonces cercana guerra

4 Consultable en: https://www.juntadeandalucia.es/cultura/redportales/cdaea/content/ colección-premio-miguel-romero-esteo\#navbar 
de los Balcanes, que pasó de ser un escenario en Combatientes, de Antonio Hernández Centeno, al argumento principal en Un lugar estratégico, de Gracia Morales. La política, desde un punto de vista crítico, ha ido ganando posiciones en la temática de estos jóvenes creadores, como refleja en distintos tonos y planos Europa Light, de Miguel Palacios, o El hombre del saco, pieza del mismo autor que navega entre un futuro no muy lejano y matices filosóficos, o El rey de Algeciras, de Juan Alberto Salvatierra, que a pesar del tiempo transcurrido continúa siendo un claro retrato de la sociedad española actual. No esquivan la historia reciente, como sucede con la pieza Españoles, Epi y Blas han muerto, de David Montero, o Franco, el retorno, de Javier Berger. Tampoco se libra de la crítica de este grupo la especulación inmobiliaria, como presenta con fino humor Sergio Rubio en Recalificados.

La situación de la mujer y el maltrato ha ido tomando fuerza con los años y convirtiéndose en una constante en algunos creadores, como Carmen Pombero, que cuenta con numerosas piezas que versan sobre aspectos poco tratados de esta lacra social, y que todas juntas componen el conjunto que la autora denomina Márgenes. Igualmente en los últimos tiempos los autores están haciendo un esfuerzo por hacer más visible con sus trabajos el tremendo problema de la migración, como sucede con Estar, llegar y quedarse, de Gracia Morales, publicada en un volumen colectivo exclusivamente dedicado a esta problemática. Es de destacar la especial sensibilidad hacia estos temas de ambas, aunque otros compañeros también se han acercado a estas realidades como José Manuel Mora en Los cuerpos perdidos, donde trata el horror de Ciudad Juárez; o Juanma Cabañas en El camino, ganadora del Premio de Teatro Mínimo Rafael Guerrero.

La crisis económica como no podía ser de otra manera, también se instaló en los textos de estos escritores, y lo hizo de dos formas posible, en la temática, como la cruda pieza Homo homini lupus, de Antonio Raposo, o la divertida, pero no menos crítica, Se alquila sofá cama, de Juan Alberto Salvatierra, o en Políticamente incorrecto, donde Tomás Afán, Javier Berger y Paco Luna, en colaboración, dan una vuelta de tuerca y hacen del teatro y la crisis, ese binomio eterno, un retrato plenamente actual de la situación de la escena. Una segunda vía fue la participación de algunos de estos creadores, como el propio Berger, en nuevas fórmulas de puesta en escena, tanto de gestión como de modelo de representación en el teatro mínimo -que no microteatro-, que propiciaba una mayor proximidad con el espectador en un entorno no convencional y con unas piezas concisas y directas.

No podemos olvidar en este apartado la relación entre este grupo de autores y los clásicos, y no solamente los españoles, que continúan siendo una fuente de inspiración de nuevas creaciones, o de interesantes versiones, como sucede con Tomás Afán, entre otros. Los clásicos contemporáneos como Bertolt Brecht, también atraen la atención de estos autores, recordemos aquí la versión realizada por José Manuel Mora de La evitable ascensión de Arturo Ui para el Centro Andaluz de Teatro en 2015. Pocos son los que han cultivado el teatro para niños, 
destacando por su dedicación y número Tomás Afán; o el teatro posdramático, el caso de Miguel Palacios, con obras como Konstante 013 o la serie PH, cuya octava entrega, PH8, les liturgies du déjeuner, fue estrenada en 2011 en el Festival d'Avignon Off.

En cuanto a la forma, la diversidad siempre ha sido amplia y enriquecedora. No obstante, hay una serie de características comunes, como la utilización de un lenguaje muy actual y poco retórico, presente en todos ellos, así como la tendencia a una escritura fragmentada, que en ocasiones también altera la línea espacial. Otra característica es el uso de la ironía y el humor, que va de la media sonrisa a la carcajada, dependiendo de la obra y el autor. La influencia del cine y la televisión es evidente desde el principio en la estructura de las escenas y las imágenes que crean, como sucede con la obra de José Francisco Ortuño Atrapados (El roto), ganadora en 1998, en la que se observan claras influencias de Quentin Tarantino, Jim Jarmusch y Woody Allen; Alguien te está mirando, de Juanma Cabañas, en el límite del guión cinematográfico y la obra dramática; o La decadencia en Varsovia, de Antonio Rojano, la cual nos puede sugerir un largo filme de carretera.

Con cierta frecuencia, las creaciones de estos autores son la plasmación de mundos irreales de creación propia, en una interesante combinación de fantasía y realidad llevada al extremo, como Elena y el fenómeno Bórvely, de Javier Berger, o en un diálogo entre un presente atemporal y un tiempo inmemorial, como Cuaderno de Bitácora, de Damaris Matos. Sin olvidar la recreación, o más bien regreso, al mundo infantil de los adultos Audrey Hepburn y Tom Waits en la obra de David Montero Lullaby. Asimismo, la combinación de tiempos y espacios es constante en algunas piezas, un buen ejemplo sería Carne de cerdo, de Pablo Gutiérrez, que transcurre en dos acciones simultáneas.

Habría que especificar que desde la primera convocatoria del premio (1997) a la última (2009), las circunstancias variaron. Hasta el 2003, los premios se convocaron anualmente; a partir de este año solamente se realizaron dos convocatorias, en 2006 y 2009. En los últimos años los concursos fueron desapareciendo, y los montajes de los textos ganadores sustituyéndose por lecturas dramatizadas. Esta variación en el funcionamiento del premio, así como los años transcurridos, incluso la diferencia de edad de los primeros premiados con los últimos, propició cierta desconexión, o menor vinculación entre los autores de la primera etapa, muy relacionados y colaboradores entre sí, y los de esta última, los ganadores Antonio Rojano (2006), Antonio Raposo Hidalgo y Antonio Rincón-Cano, ambos premiados en la última edición (2009).

En cuanto a otros autores andaluces de este periodo, como Paco Bezerra o Alberto Conejero, dramaturgos que están consiguiendo grandes éxitos en el panorama nacional, hay que aclarar que aunque comparten fechas próximas de nacimiento y unas ideas y temáticas afines, no han participado en este premio, por lo que carecen de esas experiencias y colaboración en común, ya que han desarrollado sus carreras fundamentalmente en Madrid, y sus estudios en la Real Escuela Superior de Arte Dramático de esa ciudad. 
La mayoría de los autores aquí reseñados continúan vinculados al teatro, aunque compaginan la escritura teatral con otras actividades escénicas: la escritura de guiones para televisión, como Antonio Hernández Centeno y Carmen Pombero; guiones para cine, como José Francisco Ortuño y Juanma Cabañas; la dirección de escena, como David Montero, Marilia Samper, Juan Alberto Salvatierra, Javier Berger, Miguel Palacios o Antonio Rojano; una carrera actoral, como Javier Berger; o impartiendo clases, bien en la Universidad, como Gracia Morales, escuelas de arte dramático, como Miguel Palacios, Juan Alberto Salvatierra, Rafael García Guzmán y José Manuel Mora, bien talleres en escuelas públicas y privadas de formación escénica, como David Montero. Los hay que incluso se han atrevido con la gestión cultural en alguna etapa de su vida, como Antonio Hernández Centeno, quien asumió la dirección del Teatro Maestro Álvarez Alonso, de su Martos natal, después de su inauguración en 2006. Pocos son los que han abandonado la escritura dramática, como Dámaris Matos, o Rafael Cobos, que se ha centrado exclusivamente y con gran éxito en la escritura de guiones cinematográficos. Otros, como Pablo Gutiérrez y Gonzalo Lloret Marín, están menos activos. La mayoría de ellos residen y desarrollan su carrera fundamentalmente en Andalucía, pero algunos como José Manuel Mora hace años que viven fuera de esta Comunidad, lo que ha ocasionado una mayor desconexión con el grupo.

En definitiva, este premio ha desempeñado en la dramaturgia andaluza un papel aglutinador, ha fomentado y propiciado una serie de autores dramáticos de calidad y en número considerable -que dan continuidad a otros autores andaluces de contrastada solvencia- $y$ viene a certificar el gran momento creativo por el que atraviesa esta comunidad desde los años ochenta. Según Ortega y Gasset, "Lo decisivo en la idea de las generaciones no es que se suceden, sino que se solapan o empalman. Siempre hay dos generaciones actuando al mismo tiempo... sobre los mismos temas... pero con distinto índice de edad y por ello con distinto sentido" (Ortega y Gasset 1983: 49). Nosotros nos atreveríamos a decir que, en estos momentos, en Andalucía ya ha empezado a surgir otro puñado de jóvenes creadores con gran vigor, y dispuestos a hacerse un hueco en la escena, además de seguir contando con los veteranos que continúan en activo. Se puede afirmar que al calor de este premio se formó un grupo de jóvenes autores dramáticos, los cuales comparten experiencias y características comunes, dentro de estilos bien definidos y muy diversos. Cuentan ya con largas trayectorias reconocidas, han sido representados en diferentes países, traducidos a distintos idiomas, tienen en su haber numerosos premios y comienzan a ser estudiados 5 , y todo hace pensar que pasarán a ser considerados como una generación literaria, quizás la Generación Romero Esteo.

5 Como ejemplo, la obra de Carmen Pombero es estudiada en su tesis doctoral por Ibtissam Ouadi de la Universidad de Estrasburgo; o en "Literatura" El imaginario estético de Carmen Pombero en el marco del teatro social contemporáneo", por María Rosario Naranjo Fernández de la Universidad de Sevilla. 


\section{Bibliografía}

Ambrosi. P., 2014, "La dramaturgia de Antonio Rojano", Cuadernos de Dramaturgia Contemporánea, $\mathrm{n}^{\circ} 19$.

Berger, J., 2001, Doctor, ¿es cierto que el hombre se compone de un 70\% de agua?, Sevilla, Junta de Andalucía, Consejería de Cultura, "Colección Premio Miguel Romero Esteo", núm. 0.

Centeno, A., 2001, Combatientes, Sevilla, Junta de Andalucía. Consejería de Cultura, "Colección Premio Miguel Romero Esteo", núm. 0.

Del Moral, I., 2004, "Pim, pam, clown (La guerra de los payasos) de Tomás Afán Muñoz", Las Puertas del Drama, n 19, p. 35-36.

Delgado Llergo, J., 2004, "La angustia en una pecera humana”, El Día de Córdoba, 01/07/2004.

Dilthey, W., 1994, Introducción a las ciencias del espíritu, México, Fondo de Cultura Económica.

Donaire, G., 2003, "El teatro para la infancia debe ser un contrapunto para su conciencia crítica”, El País, 07/12/2003.

Filleul, F., 2015, “Introducción”, en: Palacios, M., Europa Light. Sevilla, Junta de Andalucía. Consejería de Cultura (edición digital), Colección “Teatro en Internet", no 11, p. 4-6.

Fernández, B., 2001, "El malagueño Sergio Rubio gana el V Premio Romero Esteo de Teatro", Diario de Sevilla, 6/11/2001.

Gabriele, J. P., 2010, "Catorce voces emergentes del teatro español actual”, Anales de Literatura Española Contemporánea, vol. 35.2, año, p. 235-261.

Gambarte, M., 1996, El concepto de generación literaria, Madrid, Síntesis.

Guerrero, D., 2014, "Representar lo imposible", El Correo de Andalucía, $16 / 11 / 2014$.

Guerrero, D., 2017, “Interesante, inquietante, impactante", El Correo de Andalucía, $30 / 01 / 2017$.

Inestrosa, F., 2005, “Una tragedia contemporánea”, La Opinión, 06/11/2005.

Inestrosa, F., 2010, "Un apasionante drama humano que no busca responsables", La Opinión, 10/05/2010.

Inestrosa, F., 2010, "Las piezas de un dominó”, La Opinión,10/04/2010.

Inestrosa, F., 2013, “Compromiso en escena”, La Opinión, 05/02/2013.

Inestrosa, F., 2013, "Una excitante jornada teatral”, La Opinión, 05/05/ 2013.

Leonard, C., 2010, "Sueños de Arena by Antonio Rojano”, Estreno, vol. 36.1, p. 99-100.

Lozano, J. L., 2018, “El mar se tiñe de color gasolina”, Granada Hoy, 09/04/2018.

Martínez, L., 2003, “Todo es mentira”, ABC de Sevilla, 13/12/2003.

Martínez, L., 2010, "Javier Berger en el dormitorio de Franco", ABC de Sevilla, $19 / 02 / 2010$.

Mannheim, K., 1993, "El problema de las generaciones", Revista Española de Investigaciones Sociológicas, $\mathrm{n}^{\circ} 62$. 
Mainer, J. C., 1982, "El problema de las generaciones en la literatura española contemporánea”, Actas del Cuarto Congreso de la Asociación Internacional de Hispanistas, Salamanca, Universidad de Salamanca, p. 211-219.

Marías, J., 1949, "El método histórico de las generaciones", Revista de Occidente.

Molinari, A., 2004, "El pasado ya no tiene futuro", Ideal de Granada, 19/02/2004.

Ortega Cerpa, D., 2001, "Escribir derecho en torcido renglón”, Diario de Sevilla, 28/09/2001.

Ortega y Gasset, J., 1983, Obras completas, tomo V, Madrid, Alianza Editorial.

Ortuño, J, F., 2001, Agtrapado (El roto), Sevilla, Junta de Andalucía. Consejería de Cultura, Colección "Premio Miguel Romero Esteo", núm. 0.

Paisano, J., 2009, "Algeciras quiere poder ver su mar", Diario de Sevilla, $16 / 01 / 2009$.

Paisano, J., 2017, "Montero retrata el amor contemporáneo", Diario de Sevilla, 03/06/2017.

Palacios, M., 2007, "Influence de la recherche et des arts plastiques dans les activités de Théâtre Zéro", en: Danetis, D. (ed.), Pratiques artistiques, pratiques de recherche. Paris, l'Harmattan, p. 273-286.

Pérez-Rasilla, E., 2011, "La escritura más joven. Algunas notas sobre la escritura dramática emergente en España" Acotaciones, vol. 27, p. 13-32.

Pérez-Rasilla, E., 2012, “Notas sobre la dramaturgia emergente en España”, Don Galán (revista virtual), nº 2 .

Pérez-Rasilla, E., 2016, "Notas para un panorama del teatro español actual", Cuadernos AISPI, $\mathrm{n}^{\circ}$ 7, Teatro en España: perspectivas para el siglo XXI.

Petersen, J., 1984, "Las generaciones literarias", en: Ermatinger, E. y otros, Filosofía de la ciencia literaria. Madrid, Fondo de Cultura Económica.

Ramos, C., 2001, "La Turné, del verde al En Blanco y negro", ABC de Sevilla, $07 / 11 / 2001$.

Sadowska-Guillon, I., 2015, "Nouvelles Dramaturgies Espagnoles", Critical Stages, $\mathrm{n}^{\circ} 11$.

Sadowska-Guillon, I., 1991, "De la parole enchaînée au geste libre: La dramaturgie espagnole contemporaine depuis $1945^{\prime}$. Jeu, $\mathrm{n}^{\circ} 58$, p. 44-54.

Sánchez, R., 2014, "Compromiso social y construcción del personaje en la dramaturgia de Antonio Rincón-Cano”, en: Romera Castilla, J., Creadores jóvenes en el ámbito teatral $(20+13=33)$. Madrid, Editorial Verbum.

Scott, J. P., 2013, “Miradas ocultas”, La Opinión de Málaga, 25/02/2013.

Redacción, 2001, "El centro Andaluz de Teatro presenta el último premio Bradomín y el Romero Esteo". La Razón, Madrid, 16/10/2001.

Redacción, 2010, “Javier Berger, en el dormitorio con Franco", ABC, 19/02/2010.

T. M., 2000, "La sevillana Dámaris Matos triunfa en el Romero Esteo", Diario de Sevilla, 21/11/2000.

Zamora, A.L., 2013, “La grieta, reacciones humanas en situación de estrés”. Cienmegas. 